\title{
Metabolomics of Ginger Essential Oil against Alcoholic Fatty Liver in Mice
}

Chun-Ting Liu, ${ }^{\dagger}$ Rajasekaran Raghu, ${ }^{\dagger, \ddagger}$ Shu-Hsi Lin, ${ }^{\dagger}$ San-Yuan Wang, ${ }^{\S}$ Ching-Hua Kuo, ${ }^{\#}$ Yufeng J. Tseng, $\#, \perp, \S$ and Lee-Yan Sheen ${ }^{* \dagger,}, \mathbb{I}, \boldsymbol{\Lambda}$

${ }^{\dagger}$ Institute of Food Science and Technology, College of Bio-Resources and Agriculture, National Taiwan University, Taipei 106, Taiwan

${ }^{\ddagger}$ Department of Horticulture, College of Bio-Resources and Agriculture, National Taiwan University, Taipei 106, Taiwan

${ }^{\#}$ School of Pharmacy, College of Medicine, National Taiwan University, Taipei 106, Taiwan

${ }^{\perp}$ Graduate Institute of Biomedical Electronics and Bioinformatics, College of Electrical Engineering and Computer Science, National Taiwan University, Taipei 106, Taiwan

${ }^{\S}$ Department of Computer Science and Information Engineering, College of Electrical Engineering and Computer Science, National Taiwan University, Taipei 106, Taiwan

${ }^{\mathrm{I}}$ Center for Food and Biomolecules, College of Bio-Resources and Agriculture, National Taiwan University, Taipei 106, Taiwan

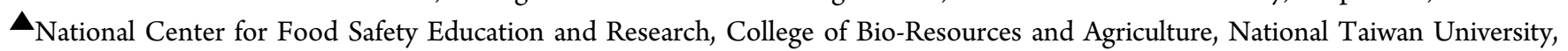
Taipei 106, Taiwan

\section{Supporting Information}

ABSTRACT: Fatty liver is significantly associated with hepatic cirrhosis and liver cancer. Excessive alcohol consumption causes alcoholic fatty liver disease (AFLD). Ginger has been reported to exhibit antioxidant potential and hepatoprotective activity. In the present study, a mouse model for AFLD was developed by employing male C57BL/6 mice that were fed an alcoholcontaining liquid diet (Lieber-DeCarli diet) ad libitum. In the treatment groups, ginger essential oil (GEO) and citral were orally administered every day for 4 weeks. Serum biochemical analysis, antioxidant enzyme activity analysis, and histopathological evaluation revealed that GEO and citral exhibited hepatoprotective activity against AFLD. Metabolites in serum samples were profiled by high-performance liquid chromatography coupled with quadrupole time-of-flight mass spectrometry (HPLC-QTOFMS). Metabolomic data indicated the amounts of metabolites such as D-glucurono-6,3-lactone, glycerol-3-phosphate, pyruvic acid, lithocholic acid, 2-pyrocatechuic acid, and prostaglandin E1 were increased after alcohol administration, but the levels were recovered in treatment groups. The analysis indicated that ginger possesses hepatoprotective properties against AFLD. Furthermore, these metabolites can serve as early noninvasive candidate biomarkers in the clinical application of AFLD for health management.

KEYWORDS: AFLD, ginger, Lieber-DeCarli diet, metabolomics, biomarker

\section{INTRODUCTION}

The liver is the largest organ in the body, and it plays important roles in metabolism. Liver diseases are mainly caused by viruses, excessive alcohol consumption, and chemicals. Repeated damage-induced inflammation causes hepatitis, hepatic fibrosis, cirrhosis, and even death due to hepatic failure. ${ }^{1-3}$

Fatty liver, a chronic liver disease, is a common global problem. Fatty liver can result in hepatitis, hepatic fibrosis, hepatic cirrhosis, liver cancer, or cardiovascular diseases. The pathogenesis of fatty liver is generally divided into nonalcoholic and alcoholic types. Excessive ethanol consumption can cause alcoholic fatty liver disease (AFLD). ${ }^{4,5}$ The mechanisms of AFLD are mediated by increased expression of cytochrome P450-2E1 (CYP2E1), ${ }^{6}$ modulation of inflammatory cytokines TNF- $\alpha$, IL- 1 , and IL- $6{ }^{7}$ and decreased hepatocyte retinoid $\mathrm{x}$ receptor levels. ${ }^{8,9}$

To analyze the relationship among alcohol, lipid, and therapeutic agents, the Lieber-DeCarli liquid diet model is frequently used to induce AFLD in animals, especially in the early stages of AFLD, including liver injury, steatosis, and oxidative stress. $^{10,11}$

Ginger (Zingiber officinale R.) and its extracts have been used widely as Chinese medicine for a long time; it has various physiological functions such as decreasing nausea, ${ }^{12}$ enhancing functions of the intestines and stomach, ${ }^{13}$ preventing cardiovascular diseases, ${ }^{14}$ promoting liver functions, ${ }^{15}$ and inhibiting cancer cell proliferation. ${ }^{16}$ Among a variety of applications, ginger essential oil (GEO) is commonly used in the food, cosmetics, and beverage industries. Notably, citral, the main constituent of GEO, has been demonstrated to exhibit antioxidant, anticancer, and anticandida effects. ${ }^{17-19}$

Metabolomics studies analyze the metabolome, which are the metabolites synthesized by a biological system and end-

Received: August 8, 2013

Revised: October 23, 2013

Accepted: October 30, 2013

Published: October 30, 2013 


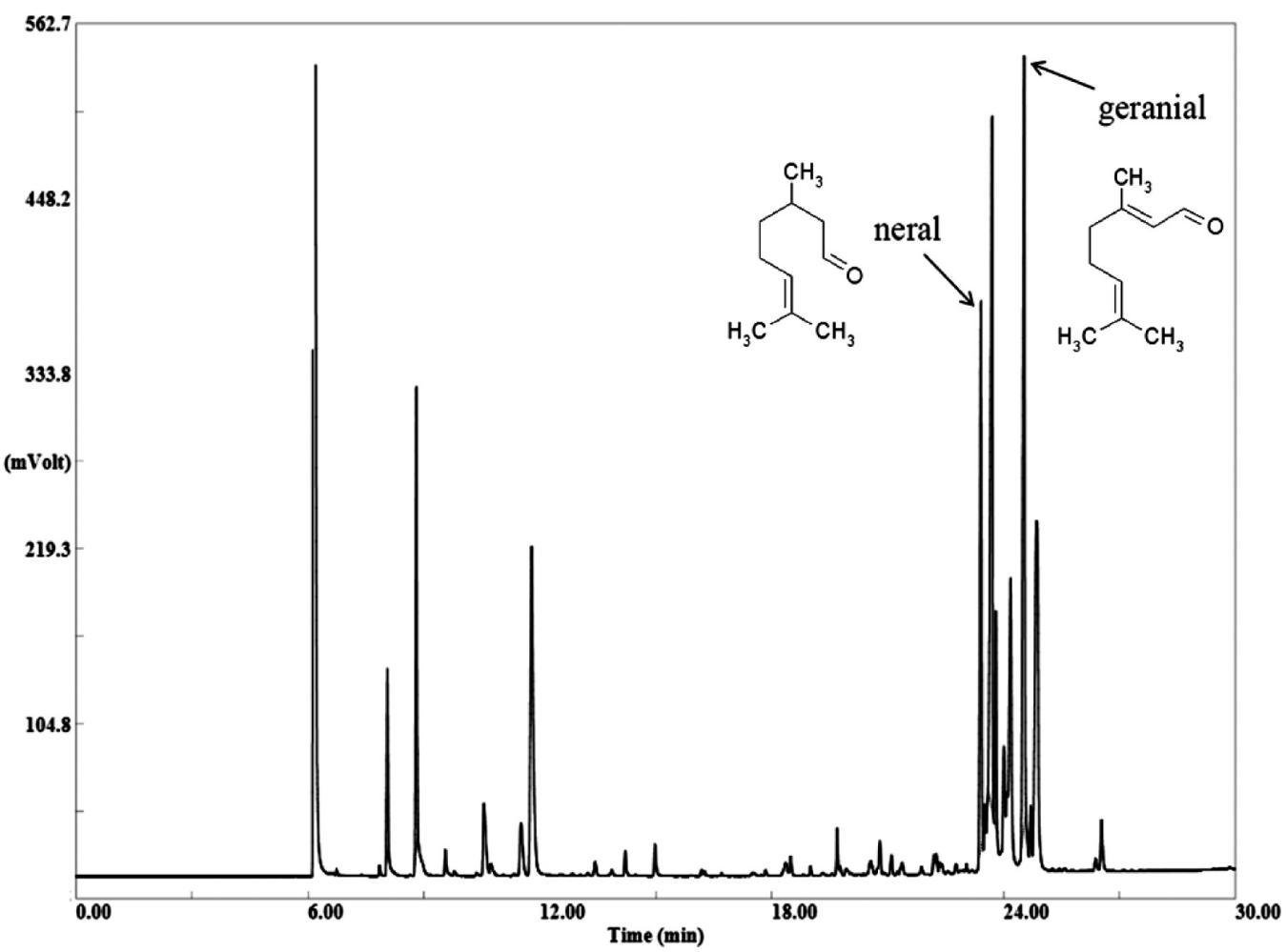

Figure 1. Volatile components of ginger essential oil (GEO).

products of gene expression in a sample. ${ }^{20,21}$ In metabolomics, blood, urine, and tissues are evaluated to generate individual metabolite profiles such as cholesterol and triglyceride profiles. In this study, we investigated the preventive effects of ginger on the formation of alcoholic fatty liver by using a metabolomics approach.

Here, we evaluated the hepatoprotective efficacy for GEO and citral by using the Lieber-DeCarli diet-induced AFLD model. Moreover, we adopted metabolomics approaches to identify differentially regulated metabolites in healthy mice and mice with AFLD. These metabolites may serve as candidate biomarkers for noninvasive detection during the early stages of AFLD, and this approach could be useful for humans.

\section{MATERIALS AND METHODS}

Chemicals. Solvents used in high-performance liquid chromatography coupled with quadrupole time-of-flight mass spectrometry (HPLC-QTOF-MS) were of MS grade. Water and acetonitrile were obtained from Scharlau (Sentmenat, Spain) and J. T. Baker (Phillipsburg, NJ, USA), respectively. Reagents were of analytical grade and were obtained from Sigma-Aldrich (Steinheim, Germany). Hexane, chloroform, and dichloromethane were procured from Merck (Darmstadt, Germany).

Preparation of Ginger Essential Oil Extracts and Citral. GEO was prepared by the steam distillation method. ${ }^{22}$ Briefly, $1 \mathrm{~kg}$ of ginger (Z. officinale $\mathrm{R}$.) was added to $3 \mathrm{~L}$ of distilled water and distilled to extract the essential oil. The constituents of GEO were analyzed by a Thermo Scientific Focus GC equipped with an AI 3000 II autosampler, a flame ionization detector, and a Stabilwax (crossbond Carbowax-PEG, Restek) column $(60 \mathrm{~m} \times 0.32 \mathrm{~mm} ; 1.0 \mu \mathrm{m})$. Standard citral (purity > 95\%) was used. The composition of GEO analyzed by gas chromatography (GC) and the representative profile (Figure 1) indicates citral (neral and geranial) as the major component in GEO (approximately 30\%). The composition of GEO was similar to previous research, ${ }^{23}$ which employed the same species of ginger used in the present study. Citral that was used for the animal experiments was obtained from Sigma-Aldrich.

In Vivo Animal Model of Alcoholic Fatty Liver Disease. Sixweek-old male C57BL/6 (B6) mice (BioLASCO Taiwan Co.) were maintained at the animal housing facility of the Institute of Food Science and Technology, National Taiwan University, at a temperature of $23 \pm 2{ }^{\circ} \mathrm{C}$ and relative humidity of $50-70 \%$ with a $12 \mathrm{~h}$ light/dark cycle. Mice were divided into a normal control group, an AFLD group, and four treatment groups. Mice in the normal control group were fed a normal liquid diet, whereas those in the AFLD and treatment groups were fed ethanol-containing Lieber-DeCarli diet. Each treatment group received either GEO [ 2.5 or $12.5 \mathrm{mg} / \mathrm{kg}$ body weight (BW)] or citral $(0.375$ or $1.875 \mathrm{mg} / \mathrm{kg} \mathrm{BW})$, both of which were mixed with olive oil (vehicle) and administered orally every day for 4 weeks. Normal and AFLD groups were given the same volume of only olive oil (vehicle). The BW of each mouse was recorded every week during the entire period of the experiment. Mice were sacrificed after 4 weeks; serum samples were then collected, and livers were sampled. Mice were handled according to the guidelines issued by the National Taiwan University Institutional Animal Care and Use Committee (Approval No. NTU-IACUC-99-53).

Serum Biochemical and Enzyme Analysis. Serum was extracted by centrifuging coagulated blood at $1000 \mathrm{~g}$ for $5 \mathrm{~min}$ at $4{ }^{\circ} \mathrm{C}$. The biochemical parameters of liver function indicators such as aspartate aminotransferase (AST), alanine aminotransferase (ALT), triglyceride (TG), and total cholesterol (TC) were estimated with commercial test strips (Commercial ALT and AST Spotchem II reagent strips, Arkray Inc., Kyoto, Japan) in an automatic blood analyzer (Spotchem EZ).

Liver Enzyme Activities. Liver homogenate was prepared by homogenizing liver $(0.5 \mathrm{~g})$ in 10 volumes of ice-cold homogenization buffer $\left(8 \mathrm{mM} \mathrm{KH}_{2} \mathrm{PO}_{4}, 12 \mathrm{mM} \mathrm{K}_{2} \mathrm{HPO}_{4}\right.$, and $\left.1.5 \% \mathrm{KCl}, \mathrm{pH} 7.4\right)$ at 4 ${ }^{\circ} \mathrm{C}$. The homogenate was then centrifuged at $10000 \mathrm{~g}$ for $30 \mathrm{~min}$ at 4 ${ }^{\circ} \mathrm{C}$. The supernatant was stored at $-80{ }^{\circ} \mathrm{C}$ and used to detect liver TG and hepatic antioxidant enzyme activity. Protein content in the homogenate was estimated spectrophotometrically by measuring the absorbance at $595 \mathrm{~nm}$ using the Bio-Rad protein Assay Kit (Hercules, CA, USA). Commercial kits purchased from the Cayman Chemical Co. (Ann Arbor, MI, USA) were used to examine the hepatic 
Table 1. Effect of Samples on Serum Biochemical and Liver Biochemical Parameters in Mice with AFLD ${ }^{a}$

\begin{tabular}{|c|c|c|c|c|c|c|}
\hline measurement & control diet & alcohol diet & $\begin{array}{c}\text { alcohol diet }+ \\
(2.5 \mathrm{mg} / \mathrm{kg} \mathrm{BW}) \\
\text { GEO }\end{array}$ & $\begin{array}{c}\text { alcohol diet }+ \\
(12.5 \mathrm{mg} / \mathrm{kg} \mathrm{BW}) \\
\text { GEO }\end{array}$ & $\begin{array}{c}\text { alcohol diet }+ \\
(0.375 \mathrm{mg} / \mathrm{kg} \mathrm{BW}) \\
\text { citral }\end{array}$ & $\begin{array}{c}\text { alcohol diet }+ \\
(1.875 \mathrm{mg} / \mathrm{kg} \mathrm{BW}) \\
\text { citral }\end{array}$ \\
\hline \multicolumn{7}{|c|}{ Serum } \\
\hline AST (IU/L) & $138 \pm 34 b$ & $185 \pm 33 a$ & $128 \pm 22 b$ & $115 \pm 42 b$ & $147 \pm 52 b$ & $120 \pm 37 b$ \\
\hline $\operatorname{ALT}(\mathrm{IU} / \mathrm{L})$ & $23 \pm 4 c$ & $65 \pm 8 \mathrm{a}$ & $39 \pm 9 b$ & $27 \pm 5 c$ & $46 \pm 18 b$ & $42 \pm 16 b$ \\
\hline cholesterol (mg/dL) & $141 \pm 15 b$ & $175 \pm 19 a$ & $122 \pm 14 c$ & $114 \pm 12 \mathrm{~cd}$ & $111 \pm 31 \mathrm{~cd}$ & $97 \pm 14 d$ \\
\hline triglyceride $(\mathrm{mg} / \mathrm{dL})$ & $99 \pm 19 b$ & $134 \pm 16 a$ & $79 \pm 25 b c$ & $57 \pm 22 \mathrm{c}$ & $92 \pm 58 b$ & $76 \pm 20 \mathrm{bc}$ \\
\hline \multicolumn{7}{|c|}{ Liver } \\
\hline triglyceride (mg/g liver) & $39 \pm 19 c$ & $91 \pm 17 a$ & $63 \pm 20 b$ & $39 \pm 21 c$ & $69 \pm 17 b$ & $65 \pm 28 b$ \\
\hline GSH (nmol/mg protein) & $38 \pm 3 b$ & $29 \pm 2 c$ & $40 \pm 3 b$ & $49 \pm 11 a$ & $38 \pm 11 b$ & $39 \pm 4 b$ \\
\hline $\mathrm{GPx}(\mathrm{nmol} / \mathrm{min} / \mathrm{mg}$ protein $)$ & $737 \pm 63 \mathrm{ab}$ & $582 \pm 51 c$ & $710 \pm 90 b$ & $812 \pm 96 a$ & $670 \pm 86 b$ & $682 \pm 42 b$ \\
\hline GRd (nmol/min/mg protein) & $34 \pm 5 a b$ & $23 \pm 6 \mathrm{~d}$ & $29 \pm 6 b c$ & $35 \pm 4 a$ & $24 \pm 3 c d$ & $29 \pm 3 b$ \\
\hline CAT $(\mathrm{nmol} / \mathrm{min} / \mathrm{mg}$ protein $)$ & $326 \pm 47 b$ & $253 \pm 18 c$ & $329 \pm 35 b$ & $395 \pm 33 a$ & $295 \pm 26 b$ & $333 \pm 55 b$ \\
\hline SOD (U/mg protein) & $52 \pm 4 b$ & $42 \pm 5 c$ & $52 \pm 4 b$ & $60 \pm 6 a$ & $50 \pm 6 b$ & $51 \pm 6 b$ \\
\hline
\end{tabular}

${ }^{a}$ Results are expressed as the mean \pm SD $(n=8)$. Statistical analysis was performed among treatments within each group. Different letters $(a-d)$ indicate a significant difference among groups according to the one-way ANOVA coupled with the Duncan's multiple-comparison test $(p<0.05)$. AFLD, alcoholic fatty liver disease; GEO, ginger essential oil; AST, aspartate aminotransferase; ALT, alanine aminotransferase; GSH, glutathione; GPx, glutathione peroxidase; GRd, glutathione reductase; CAT, catalase; SOD, superoxide dismutase.

antioxidant systems of glutathione (GSH; item 703002), glutathione peroxidase (GPx; item 703102), glutathione reductase (GRd; item 703202), catalase (CAT; item 707002), and superoxide dismutase (SOD; item 706002).

Liver Biopsy Examination. Liver histological sections for pathological staining and semiquantitative analysis were performed from the right lobe of the liver to avoid observational bias. For histopathological observations, formalin-fixed paraffin-embedded (FFPE) liver sections were observed for liver fatty accumulation, necrosis, fibrosis, and other changes in liver tissues, after staining with hematoxylin and eosin (H\&E). Scoring and interpretation of biopsy results were carried out by an experienced pathologist, College of Veterinary Medicine Animal Disease Diagnostic Center, National Chung Hsing University, Taichung, Taiwan.

Metabolic Profiling. Sample Preparation. Aliquots of $100 \mu \mathrm{L}$ of serum were added to extraction solvents $(1: 4)$ and extracted by the Geno Grinder 2010 (SPEX, Pittsburg, CA, USA) at $1000 \mathrm{rpm}$ for 2 min. Extracts were centrifuged at $15000 \mathrm{~g}$ for $5 \mathrm{~min}$ at $4{ }^{\circ} \mathrm{C}$. The supernatant was collected and evaporated by a centrifugal vaporizer (EYELA, Tokyo, Japan) for $2 \mathrm{~h}$. The residue was reconstituted in 200 $\mu \mathrm{L}$ of $50 \%$ methanol and centrifuged at $15000 \mathrm{~g}$ for $5 \mathrm{~min}$. The supernatant was filtered with a $0.2 \mu \mathrm{m}$ Minisart RC 4 filter (Sartorius, Goettingen, Germany) and subjected to HPLC-QTOF-MS analysis. The same amounts of sample aliquots were pooled for the QC sample.

Chromatographic and Mass Spectrometric Analysis. All serum samples were analyzed by an Agilent 1290 U-HPLC system coupled with 6540-QTOF (Agilent Technologies, Santa Clara, CA, USA). The sample $(2 \mu \mathrm{L})$ was injected into an ACQUITY UPLC HSS T3 column $(2.1 \times 100 \mathrm{~mm} ; 1.8 \mu \mathrm{m})$ (Waters, Milford, MA, USA). The mobile phase consisted of $0.1 \%$ formic acid (solvent A) and acetonitrile (solvent B). The gradient profile used was as follows: $0-1.5 \mathrm{~min}, 2 \%$ B; 1.5-9 min, linear gradient from 2 to $50 \%$ B; 9-14 min, linear gradient from $50 \%$ to $95 \% \mathrm{~B} ; 14-15 \mathrm{~min}, 95 \% \mathrm{~B}$; the column was then re-equilibrated. The flow rate was maintained at $0.3 \mathrm{~mL} / \mathrm{min}$. The column oven was set at $40{ }^{\circ} \mathrm{C}$, and the autoinjection system was set at $10^{\circ} \mathrm{C}$. A Jet Stream electrospray ion source with capillary voltage of 4 $\mathrm{kV}$ in positive mode and $3.5 \mathrm{kV}$ in negative mode was used for sample ionization. MS parameters were set as follows: dry gas temperature, $325{ }^{\circ} \mathrm{C}$; dry gas flow, $5 \mathrm{~L} / \mathrm{min}$; nebulizer, $40 \mathrm{psi}$; sheath gas temperature, $325{ }^{\circ} \mathrm{C}$; sheath gas flow, $10 \mathrm{~L} / \mathrm{min}$; and fragmentor, 120 $\mathrm{V}$. The scan range was $m / z 50-1700$.

Data Analysis. The Kyoto Encyclopedia of Genes and Genomes (KEGG) database was used to identify metabolite biomarkers by comparing molecular weights. The molecular structures of candidate compounds were retrieved by comparison and then confirmed by MS scans for characteristic ions and fragmentation patterns of metabolites.
Three-dimensional principal component analysis (3D-PCA) was performed, and the values of PC1, PC2, and PC3 were plotted with designation of cases as control, AFLD, or treatment groups.

Statistical Analysis. Statistical differences between groups were compared by one-way analysis of ANOVA software with Duncan's multiple-comparison test by SPSS software (Statistical Package for Social Sciences, SPSS Corporation, Chicago, IL, USA). All values are expressed as the mean $\pm \mathrm{SD}$.

\section{RESULTS AND DISCUSSION}

In Vivo Model for Alcohol-Induced Fatty Liver. Establishing an ideal rodent model for the study of alcoholic fatty liver is a prerequisite for this experiment. Because metabolic rates considerably differ among species, optimization and validation of the model are necessary. In our analysis, C57BL/6 mice were employed to develop a model for alcoholic fatty liver using the Leiber-DeCarli liquid diet. The ability to induce visible phenotypic symptoms of alcohol-induced liver disease such as steatosis, inflammation, and necrosis is a benchmark for the model's success. Many studies have failed to induce hepatitis coupled with fatty liver. ${ }^{24,25}$ However, consumption of alcohol-containing Leiber-DeCarli liquid diet for 28 days has been shown to result in alcoholic fatty liver, inflammation, and necrosis in C57BL/6 mice. ${ }^{26}$

Hepatoprotective Activity of Ginger on AFLD. Here, we induced similar effects in C57BL/6 mice after feeding for 28 days. The AFLD group recorded a significant $(p<0.05)$ increase in the serum AST and ALT activity levels after 28 days of feeding, whereas mice fed control Leiber-DeCarli liquid diet (normal control group) exhibited no liver damage. The AFLD group exhibited a significant $(p<0.05)$ increase in TC and TG levels when compared to the normal control group. Moreover, AST, ALT, TC, and TG levels were recovered significantly in the treatment groups compared to the AFLD group $(p<0.05)$, especially in mice gavaged with high doses of GEO (Table 1). GSH is an important indicator of the liver antioxidant system. GSH levels were significantly decreased in the AFLD group compared to the normal control group. Similarly, liver antioxidant enzyme activities, namely, GPx, GRd, catalase, and SOD, were lower in the AFLD group than in the control group. However, a significant increase was observed in the antioxidant activity of these enzymes in the treatment groups ( $p$ 
Table 2. Histopathological Analysis for the Fatty Liver ${ }^{a}$

\begin{tabular}{|c|c|c|c|c|c|c|}
\hline score & control diet & alcohol diet & $\begin{array}{c}\text { alcohol diet }+(2.5 \mathrm{mg} / \mathrm{kg} \\
\text { BW }) \text { GEO }\end{array}$ & $\begin{array}{c}\text { alcohol diet }+(12.5 \mathrm{mg} / \mathrm{kg} \\
\text { BW }) \text { GEO }\end{array}$ & $\begin{array}{c}\text { alcohol diet }+(0.375 \mathrm{mg} / \mathrm{kg} \\
\text { BW }) \text { citral }\end{array}$ & $\begin{array}{c}\text { alcohol diet }+(1.875 \mathrm{mg} / \mathrm{kg} \\
\text { BW }) \text { citral }\end{array}$ \\
\hline fatty liver & $1.0 \pm 0.5 c$ & $3.5 \pm 0.5 a$ & $2.0 \pm 0.8 b$ & $1.3 \pm 0.7 c$ & $2.8 \pm 1.0 \mathrm{~b}$ & $2.3 \pm 0.7 b$ \\
\hline
\end{tabular}

${ }^{a}$ Results are expressed as the mean \pm SD $(n=8)$. Statistical analysis was performed among treatments within each group. Different letters (a-c) indicate a significant difference among groups according to the one-way ANOVA coupled with the Duncan's multiple-comparison test $(p<0.05)$. GEO, ginger essential oil. Degree of lesions was graded from 1 to 5 depending on severity: $1=$ minimal $(<1 \%) ; 2=$ slight $(1-25 \%) ; 3=$ moderate $(26-50 \%) ; 4=$ moderate/severe $(51-75 \%) ; 5$ = severe/high $(76-100 \%)$.
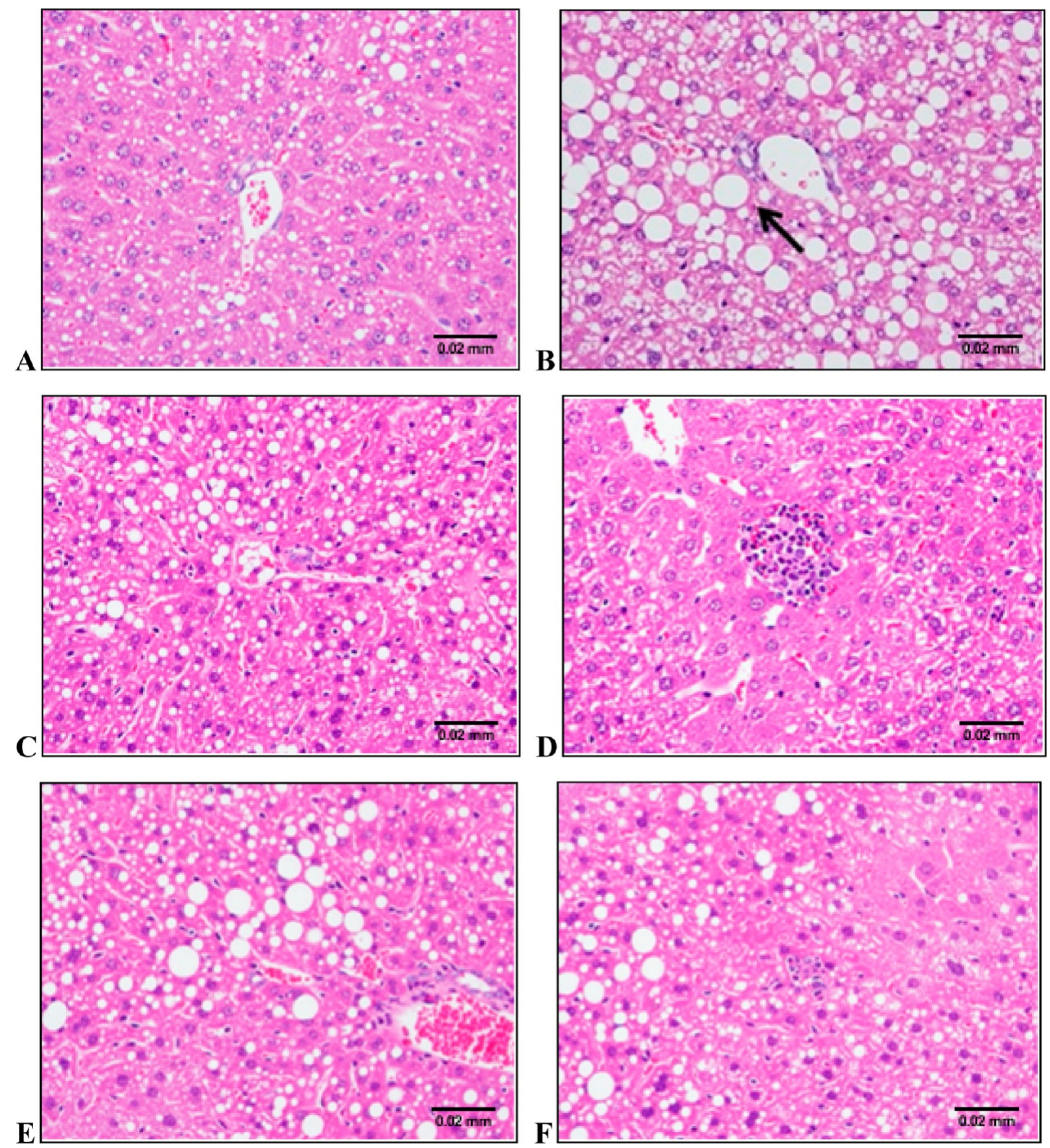

Figure 2. Representative histological features of liver section stained with H\&E (400X) depicting the effect of samples on liver histopathology of mice with AFLD. Control liquid diet induced minimal (1) fatty infiltration with macrovesicles of hepatocytes in the normal control group (A). Alcohol liquid diet-induced fatty liver with macrovesicles (indicated by arrowhead) of hepatocytes was graded as moderate in AFLD group (B); minimal (1) in high dose of GEO (12.5 mg/kg BW) group (D), and slight (2) in low dose of GEO (2.5 mg/kg BW) (C), low dose of citral (0.375 $\mathrm{mg} / \mathrm{kg} \mathrm{BW})(\mathrm{E})$, and high dose of citral $(1.875 \mathrm{mg} / \mathrm{kg} \mathrm{BW})$ groups $(\mathrm{F})$. 


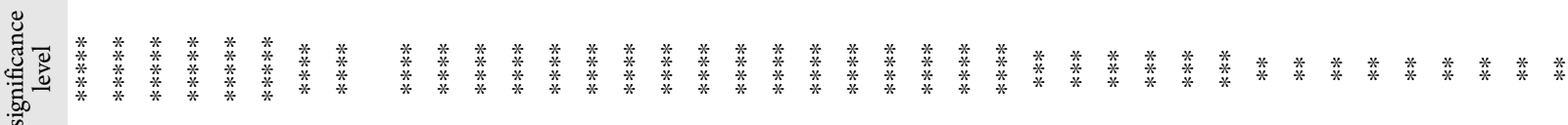
W س 月 U m

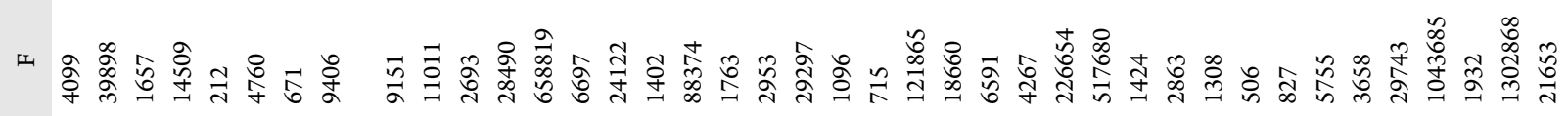

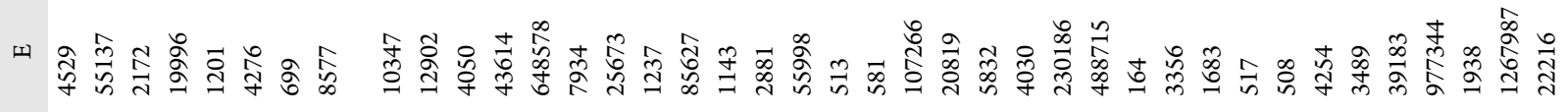

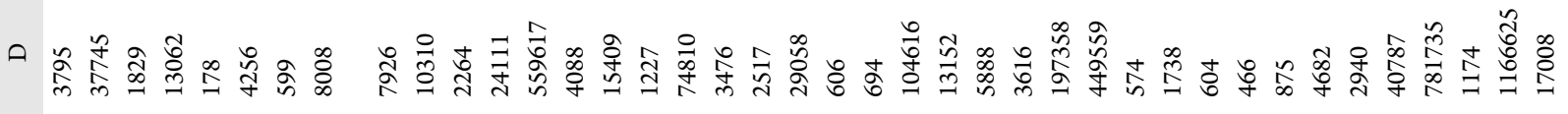

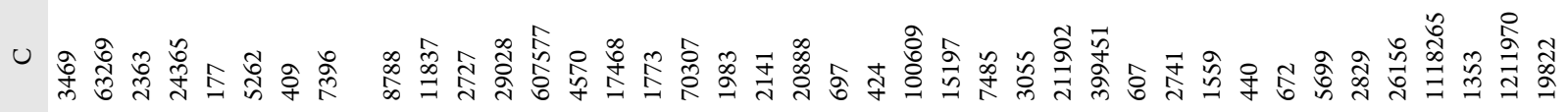

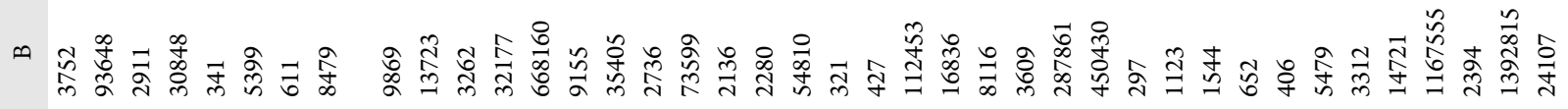

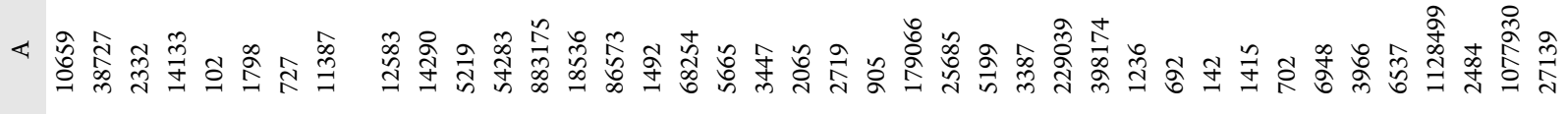
N

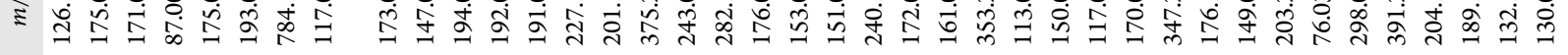




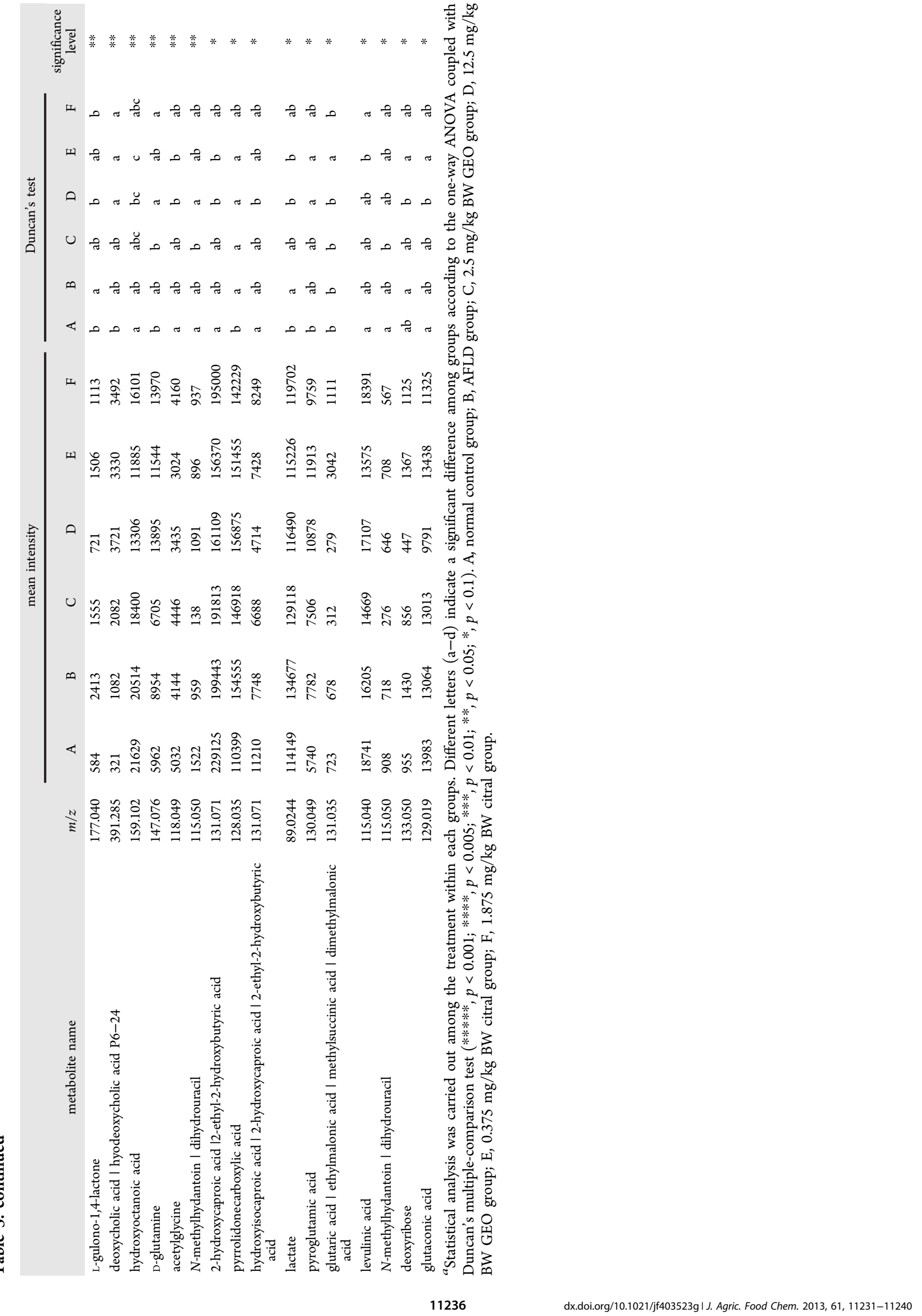


$<0.05$; Table 1) compared to the AFLD group. It has been reported that ginger ameliorates fatty liver and hypertriglyceridemia in rats. ${ }^{27}$ In addition, results of other studies have confirmed that ginger exerts hepatoprotective properties against liver damage by bromobenzene, acetaminophen, carbon tetrachloride, and ethanol. ${ }^{28,29}$ In the present study, the hepatoprotective activity of ginger on AFLD was found to be similar to that reported previously. Histopathological observation of liver sections from the control group exhibited a normal liver architecture, whereas fatty infiltration with macrovesicles was observed in the livers of the AFLD group. However, compared to the AFLD group, there was a significant improvement in treatment groups gavaged with a high dosage of GEO or citral (Table 2; Figure 2). Recently, more research has focused on herbal remedies for alcoholic liver diseases. ${ }^{30}$ Garlic and ginger are familiar, traditional Chinese herbs; it has been reported that garlic exhibits liver protection properties in alcohol-induced fatty liver. ${ }^{31}$ Our study results show that ginger provided hepatoprotection to alcohol-induced fatty liver. Hence, ginger may be a potential hepatoprotective candidate against AFLD.

Metabolic Profiling of Serum Samples of AFLD. Metabolomes of serum samples from control, AFLD, and treatment groups were profiled by U-HPLC/Q-TOF MS. The metabolites with a significant difference among groups are listed in Table 3. We investigated the overall metabolic differences by using the 3D-PCA method. 3D-PCA was used as an unsupervised statistical method to study the metabolome differences between AFLD, treatment mice, and healthy controls. The score plots of 3D-PCA are displayed in Figure 3. Results revealed that $\mathrm{PC} 1$ accounted for $54 \%$ of variations in the 3D-PCA model, PC2 for 22\%, and PC3 for $15 \%$; these extracts clearly enabled differentiation. These separation results indicate the potential diagnostic value of metabolic profiles that reflect biochemical differences among the control, AFLD, and treatment groups. A one-way ANOVA followed by Duncan's

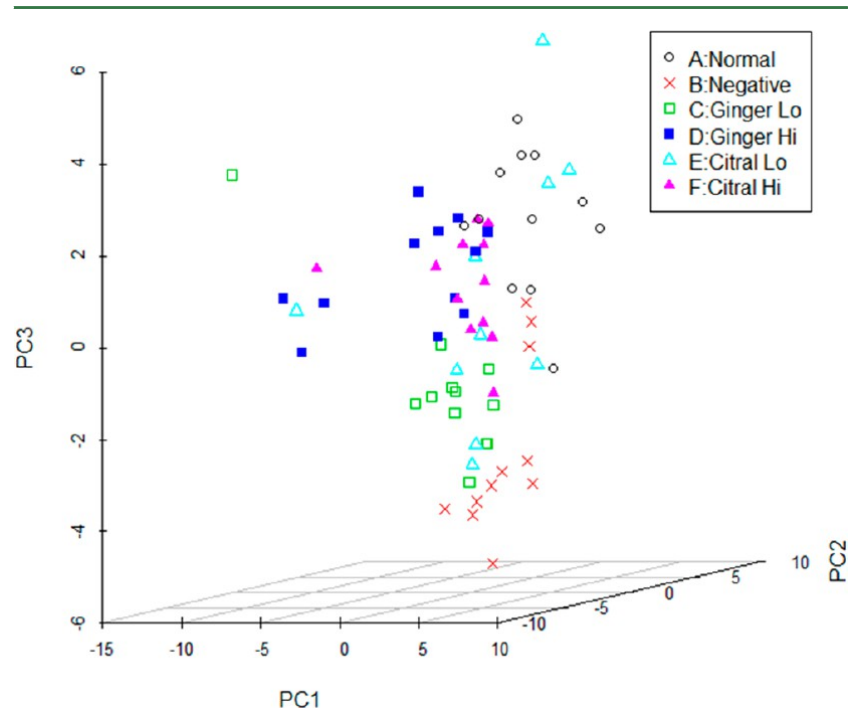

Figure 3. Three-dimensional principal component analysis (3D-PCA) of serum samples obtained from the control, AFLD, and treatment mice. PC1 is $54 \%, \mathrm{PC} 2$ is $22 \%, \mathrm{PC} 3$ is $15 \%$. (Open black circles): A, normal control group; (red times signs) B, AFLD group; (open green squares) C, $2.5 \mathrm{mg} / \mathrm{kg}$ BW GEO group; (solid blue squares) D, 12.5 $\mathrm{mg} / \mathrm{kg}$ BW GEO group; (open teal triangles) E, $0.375 \mathrm{mg} / \mathrm{kg}$ BW citral group; (solid pink triangles) F, $1.875 \mathrm{mg} / \mathrm{kg}$ BW citral group. multiple-comparison test was used to analyze the statistically significant differences of metabolites among the control, AFLD, and treatment groups. Levels of D-glucurono-6,3-lactone, glycerol-3-phosphate, and pyruvic acid, which are important metabolites in many metabolic pathways, were significantly increased $(p<0.001)$ in the AFLD group compared to those in the normal control group (Figure 4). However, levels of these metabolites were significantly decreased $(p<0.001)$ in the treatment groups compared to the levels in the AFLD group. Similar changes in the levels of other metabolites involved in key metabolic pathways, including lithocholic acid $(p<0.005)$, 2 -pyrocatechuic acid $(p<0.005)$, prostaglandin E1 $(p<0.005)$, methionine $(p<0.01)$, L-gulono-1,4-lactone $(p<0.05)$, and lactate $(p<0.1$; Figures 5 and 6), were observed upon treatment (Figure 4-6). The levels of these metabolites in the AFLD group were the highest among the six groups of mice, but these metabolites were down-regulated in the treatment groups. Our results suggest that these metabolites are related to AFLD. These metabolites can be used as potential candidate biomarkers for healthy management and clinical application after they are validated in humans.

After ingestion, alcohol is metabolized to acetaldehyde by $\mathrm{ADH}$, the microsomal ethanol-oxidizing enzyme CYP2E1, and bacterial enzymes of intestinal microbiota. Acetaldehyde is then oxidized to acetate by ALDH and introduced into the citric acid cycle (Krebs cycle) as acetyl-CoA. Alcohol metabolism is known to significantly slow the TCA cycle when fatty acids are available in mice ${ }^{26}$ and rats. ${ }^{32} \mathrm{We}$ observed changes in the metabolite lactate, which is consistent with the data presented by Bradford et al. Higher levels of lactate correlate with hypoxia. ${ }^{33}$ Chronic alcohol administration increases the rate of alcohol metabolism, ${ }^{34}$ induces oxidative stress, the production of reactive oxygen species (ROS), cytokine release, mitochondrial dysfunction, and endoplasmic reticulum stress. Alcohol also stimulates liver metabolism and increases oxygen uptake, leading to hypoxia in the pericentral regions in liver lobules. ${ }^{35}$ ROS initiate lipid peroxidation that directly damages plasma and intracellular membranes and leads to the production of reactive aldehydes with potent pro-inflammatory and profibrotic properties. Acute alcohol exposure results in increased ketogenesis and increased lipid peroxidation. ${ }^{36}$ Chronic treatment of liver with alcohol induces liver inflammation through key arachidonic acid pathway metabolites including prostaglandins E1, prostacyclins, thromboxanes, and leukotrienes.

Liver injury also influences phenylalanine metabolism by affecting the following metabolites: succinate, pyruvate, and 2hydroxyphenylacetate. An alcohol diet also affects arginine and proline metabolism. In addition, major amino acid metabolism pathways, including phenylalanine metabolism, glycine, serine, and threonine metabolism, arginine and proline metabolism, tryptophan metabolism, and valine, leucine, and isoleucine biosynthesis, as well as alanine, aspartate, and glutamate metabolism, are influenced by alcohol administration. All of these are inter-related with fatty acid, nucleotide, and carbohydrate metabolism. Phenylalanine, tyrosine, leucine, and tryptophan have been considered serum biomarkers for liver injury induced by alcohol. ${ }^{37}$ In this study, we found that all pathways involving metabolites were influenced by alcohol administration. However, plasma lipid metabolic profiling can give a reasonable understanding of the pathogenesis of ethanolmediated liver injury. ${ }^{38}$ Furthermore, L-acetylcarnitine concentration was increased in the sera of humans with liver cirrhosis compared with that of healthy subjects. ${ }^{39}$ Our results show that 

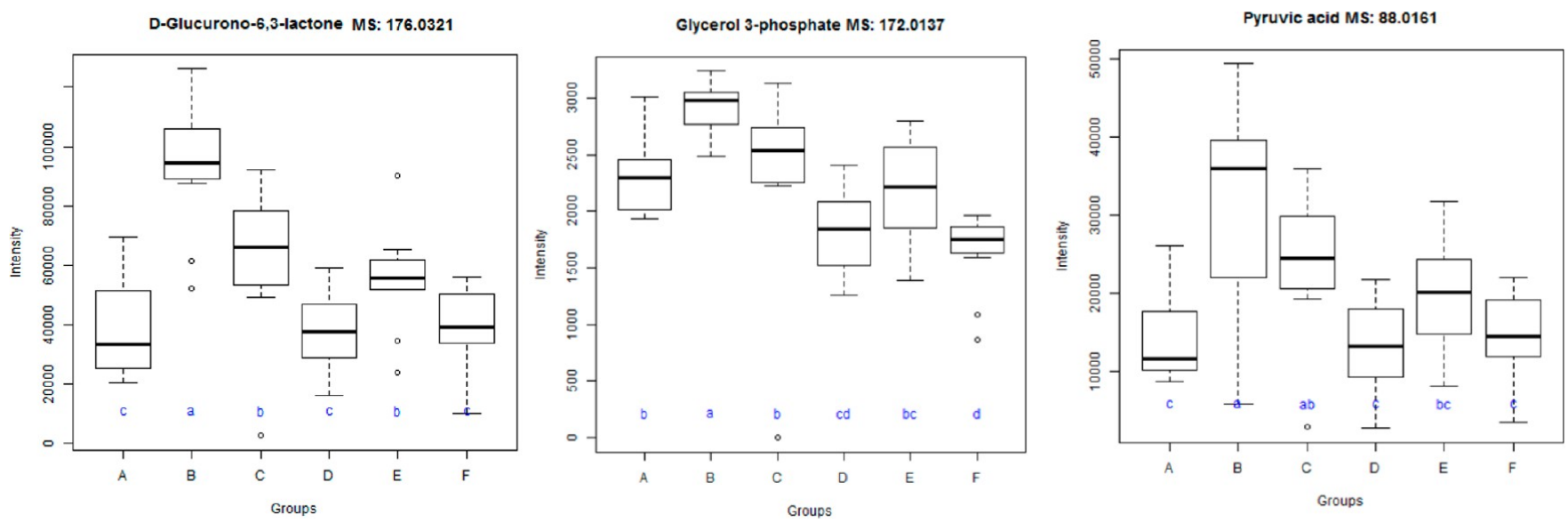

Figure 4. Metabolites of mice with AFLD that changed significantly among the six groups of mice (D-glucurono-6,3-lactone, glycerol-3-phosphate, and pyruvic acid; $p<0.001$ ): A, normal control group; B, AFLD group; C, $2.5 \mathrm{mg} / \mathrm{kg}$ BW GEO group; D, $12.5 \mathrm{mg} / \mathrm{kg}$ BW GEO group; E, 0.375 $\mathrm{mg} / \mathrm{kg} \mathrm{BW}$ citral group; F, $1.875 \mathrm{mg} / \mathrm{kg}$ BW citral group. Statistical analysis was performed among treatments within each group. Different letters $(\mathrm{a}-\mathrm{d})$ indicate a significant difference among groups according to one-way ANOVA coupled with the Duncan's multiple-comparison test $(p<$ $0.001)$.
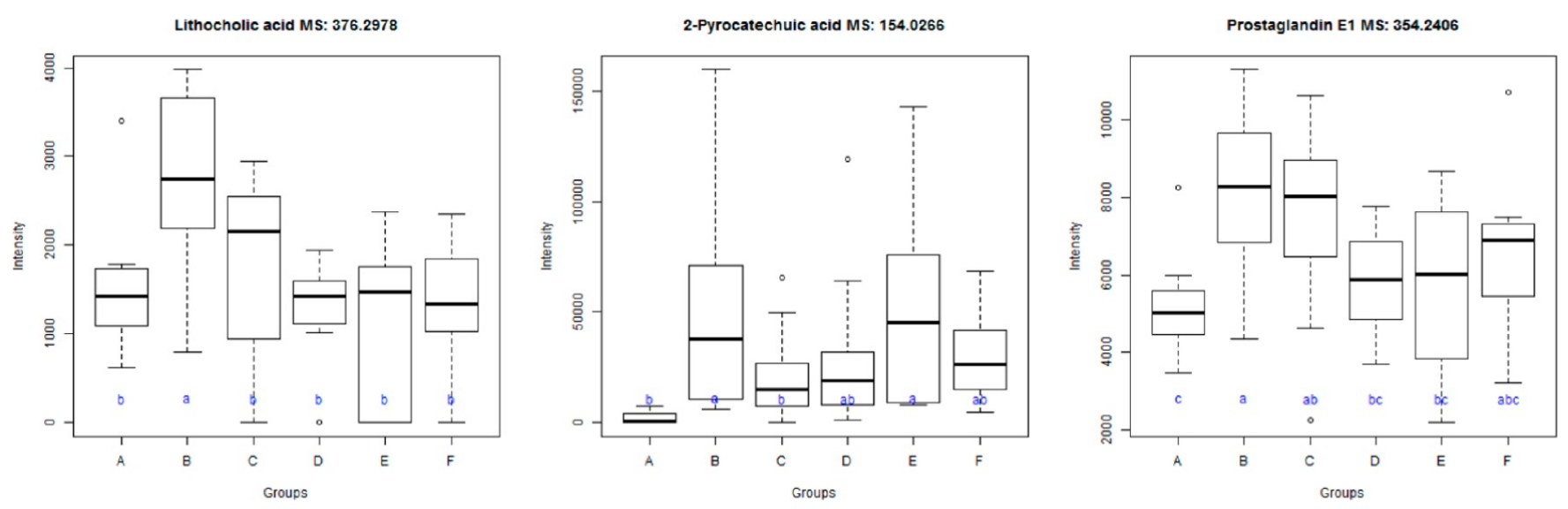

Figure 5. Metabolites of mice with AFLD that changed significantly among the six groups of mice (lithocholic acid, 2-pyrocatechuic acid, and prostaglandin E1; $p<0.005$ ): A, normal control group; B, AFLD group; C, $2.5 \mathrm{mg} / \mathrm{kg}$ BW GEO group; D, $12.5 \mathrm{mg} / \mathrm{kg}$ BW GEO group; E, 0.375 $\mathrm{mg} / \mathrm{kg} \mathrm{BW}$ citral group; F, $1.875 \mathrm{mg} / \mathrm{kg}$ BW citral group. Statistical analysis was performed among the treatment within each group. Different letters $(\mathrm{a}-\mathrm{c})$ indicate a significant difference among groups according to one-way ANOVA coupled with the Duncan's multiple-comparison test $(p<$ $0.005)$.
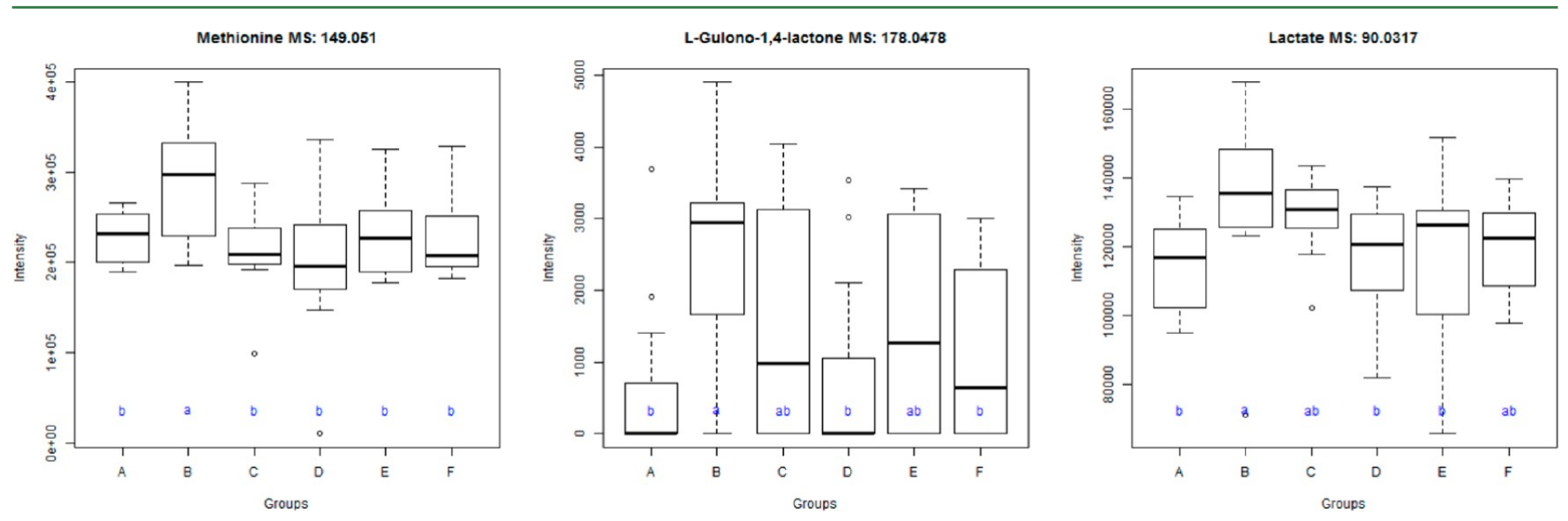

Figure 6. Metabolites of mice with AFLD that changed significantly among the six groups of mice (methionine, L-gulono-1,4-lactone, and lactate; $p<$ $0.01, p<0.05, p<0.1$, respectively): A, normal control group; B, AFLD group; C, $2.5 \mathrm{mg} / \mathrm{kg} \mathrm{BW}$ GEO group; D, $12.5 \mathrm{mg} / \mathrm{kg} \mathrm{BW} \mathrm{GEO} \mathrm{group;} \mathrm{E,}$ $0.375 \mathrm{mg} / \mathrm{kg}$ BW citral group; F, $1.875 \mathrm{mg} / \mathrm{kg}$ BW citral group. Statistical analysis was performed among treatments within each group. Different letters $(\mathrm{a}, \mathrm{b})$ indicate a significant difference among groups according to one-way ANOVA coupled with the Duncan's multiple-comparison test. 
the levels of fatty liver induced by consumption of an alcoholic liquid diet were not significant, which may have led to similar concentrations of L-acetylcarnitine in the control and AFLD groups.

Metabolomics results showed changes in the levels of Dglucurono-6,3-lactone and L-gulono-1,4-lactone, which are key metabolites in ascorbate and aldarate metabolism. Moreover, glucuronic acid exhibits hepatoprotective properties in liver detoxification systems, and it metabolizes to glucuronolactone in the liver. Lithocholic acid also plays an important role in bile secretion, which is involved in alcohol-induced liver damage. ${ }^{40}$ In our research, alcohol consumption induced an increase in the $\mathrm{NADH} / \mathrm{NAD}^{+}$ratio. It also increased the activity of glycerophosphate dehydrogenase and promoted the conversion of dihydroxyacetone phosphate to glycerol-3-phosphate, which might enhance lipid synthesis in the liver. In our study, 2pyrocatechuic acid was detected in the metabolites, which could serve as a biological marker for the detection and quantification of $\mathrm{OH}$ radicals. ${ }^{41}$ The levels of essential amino acids such as leucine, isoleucine, and methionine were elevated in the control, AFLD, and treatment groups; this finding was consisted with the result of a previous study, ${ }^{42}$ which suggests a higher rate of whole-body protein turnover. In addition, methionine is involved in many important pathways such as cysteine and methionine metabolism, protein digestion and absorption, aminoacyl-tRNA biosynthesis, and mineral absorption. Although hepatic steatosis could be observed with the noninvasive magnetic resonance imaging (MRI) technique, liver inflammation could not be detected using this technique. ${ }^{43}$ Variations of these metabolites, which play very important roles in physiological metabolism in the body, could be used as noninvasive biomarkers of AFLD.

Conclusion. Ginger exhibited hepatoprotective properties to prevent AFLD. In particular, GEO played an important role in hepatoprotection. The metabolomic data obtained in this study provide new, significant mechanistic clues concerning the effects of an alcoholic liquid diet on liver metabolism, which may be crucial for understanding the pathogenesis of alcoholinduced liver disease. We propose that several metabolites could be used as novel noninvasive biomarkers of AFLD induced by alcohol consumption and oxidative stress. Furthermore, these metabolites observed in mice with AFLD need to be compared and validated in human samples to reveal their potential as biomarkers.

\section{ASSOCIATED CONTENT}

\section{S Supporting Information}

Additional table. This material is available free of charge via the Internet at http://pubs.acs.org.

\section{AUTHOR INFORMATION}

\section{Corresponding Author}

*(L.-Y.S.) Mailing address: No. 1, Section 4, Roosevelt Road, Taipei 106, Taiwan. Phone: 886-2-33664129. Fax: 886-223620849. E-mail: lysheen@ntu.edu.tw.

\section{Funding}

This research work was funded in part by the National Science Council (NSC-99-2321-B-002-042) and National Taiwan University (Aim For Top University Program 102R-7620), Taiwan.

\section{Notes}

The authors declare no competing financial interest.

\section{ACKNOWLEDGMENTS}

We thank Prof. Shu-Chen Hsieh for helpful discussions in experimental design. We also thank Dr. Guan-Yuan Chen, Mr. Jia-Wei Liu, and Miss Wei-Ting Dai for technical support. We thank the NTU Integrated Core Facility for Functional Genomics of National Research Program for Genomic Medicine of Taiwan for technical assistance.

\section{ABBREVIATIONS USED}

AFLD, alcoholic fatty liver disease; GEO, ginger essential oil; HPLC-QTOF-MS, high-performance liquid chromatography coupled with quadrupole time-of-flight mass spectrometry; AST, aspartate aminotransferase; ALT, alanine aminotransferase; TG, triglyceride; TC, total cholesterol; GSH, glutathione; GPx, glutathione peroxidase; GRd, glutathione reductase; CAT, catalase; SOD, superoxide dismutase; $\mathrm{ADH}$, alcohol dehydrogenase; ALDH, aldehyde dehydrogenase; ROS, reactive oxygen species; 3D-PCA, three-dimensional principal component analysis; MRI, magnetic resonance imaging

\section{REFERENCES}

(1) Albano, E. Alcohol, oxidative stress and free radical damage. Proc. Nutr. Soc. 2006, 65, 278-290.

(2) Hoek, J. B.; Pastorino, J. G. Ethanol, oxidative stress, and cytokine-induced liver cell injury. Alcohol 2002, 27, 63-68.

(3) Jaeschke, H. Reactive oxygen and mechanisms of inflammatory liver injury. J. Gastroenterol. Hepatol. 2000, 15, 718-724.

(4) Liu, J. D.; Leung, K. W.; Wang, C. K.; Liao, L. Y.; Wang, C. S.; Chen, P. H.; Chen, C. C.; Yeh, E. K. Alcohol-related problems in Taiwan with particular emphasis on alcoholic liver diseases. Alcohol.: Clin. Exp. Res. 1998, 22, 164S-169S.

(5) Stewart, S.; Jones, D.; Day, C. P. Alcoholic liver disease: new insights into mechanisms and preventative strategies. Trends Mol. Med. 2001, 7, 408-413.

(6) Lu, Y.; Cederbaum, A. I. CYP2E1 and oxidative liver injury by alcohol. Free Radical Biol. Med. 2008, 44, 723-738.

(7) Mandrekar, P.; Szabo, G. Signalling pathways in alcohol-induced liver inflammation. J. Hepatol. 2009, 50, 1258-1266.

(8) Dai, T.; Wu, Y.; Leng, A. S.; Ao, Y.; Robel, R. C.; Lu, S. C.; French, S. W.; Wan, Y. J. RXR $\alpha$-regulated liver SAMe and GSH levels influence susceptibility to alcohol-induced hepatotoxicity. Exp. Mol. Pathol. 2003, 75, 194-200.

(9) Gyamfi, M. A.; He, L.; French, W.; Damjanov, I.; Wan, Y. J. Hepatocyte retinoid $\mathrm{X}$ receptor $\alpha$-dependent regulation of lipid homeostasis and inflammatory cytokine expression contributes to alcohol-induced liver injury. J. Pharmacol. Exp. Ther. 2008, 324, 443453.

(10) Lieber, C. S.; DeCarli, L. M. Liquid diet technique of ethanol administration: 1989 update. Alcohol Alcohol. (Oxford, U.K.) 1989, 24, 197-211.

(11) Lieber, C. S.; DeCarli, L. M.; Sorrell, M. F. Experimental methods of ethanol administration. Hepatology 1989, 10, 501-510.

(12) Vutyavanich, T.; Kraisarin, T.; Ruangsri, R. Ginger for nausea and vomiting in pregnancy: randomized, double-masked, placebocontrolled trial. Obstet. Gynecol. 2001, 97, 577-582.

(13) Micklefield, G. H.; Redeker, Y.; Meister, V.; Jung, O.; Greving, I.; May, B. Effects of ginger on gastroduodenal motility. Int. J. Clin. Pharmacol. Ther. 1999, 37, 341-346.

(14) Fuhrman, B.; Rosenblat, M.; Hayek, T.; Coleman, R.; Aviram, M. Ginger extract consumption reduces plasma cholesterol inhibits LDL oxidation and attenuates development of atherosclerosis in atherosclerotic, apolipoprotein E-deficient mice. J. Nutr. 2000, 130, $1124-1131$.

(15) Matsuda, A.; Wang, Z.; Takahashi, S.; Tokuda, T.; Miura, N.; Hasegawa, J. Upregulation of mRNA of retinoid binding protein and 
fatty acid binding protein by cholesterol enriched-diet and effect of ginger on lipid metabolism. Life Sci. 2009, 84, 903-907.

(16) Choudhury, D.; Das, A.; Bhattacharya, A.; Chakrabarti, G. Aqueous extract of ginger shows antiproliferative activity through disruption of microtubule network of cancer cells. Food Chem. Toxicol. 2010, 48, 2872-2880.

(17) Nakamura, Y.; Miyamoto, M.; Murakami, A.; Ohigashi, H.; Osawa, T.; Uchida, K. A phase II detoxification enzyme inducer from lemongrass identification of citral and involvement of electrophilic reaction in the enzyme induction. Biochem. Biophys. Res. Commun. 2003, 302, 593-600.

(18) Xia, H.; Liang, W.; Sonq, Q.; Chen, X.; Chen, X.; Hong, J. The in vitro study of apoptosis in NB4 cell induced by citral. Cytotechnology 2013, 65, 49-57.

(19) Takahashi, M.; Inouye, S.; Abe, S. Anti-Candida and radical scavenging activities of essential oils and oleoresins of Zingiber officinale Roscoe and essential oils of other plants belonging to the family Zingiberaceae. Drug Discov. Ther. 2011, 5, 238-245.

(20) Corthesy-Theulaz, I.; den Dunnen, J. T.; Ferre, P.; Geurts, J. M. W.; Muller, M.; van Belzen, N.; van Ommen, B. Nutrigenomics: the impact of biomics technology on nutrition research. Ann. Nutr. Metab. 2005, 49, 355-365.

(21) Gibney, M. J.; Walsh, M.; Brennan, L.; Roche, H. M.; German, B.; van Ommen, B. Metabolomics in human nutrition: opportunities and challenges. Am. J. Clin. Nutr. 2005, 82, 497-503.

(22) Chen, H. W.; Yang, J. J.; Tsai, C. W.; Wu, J. J.; Sheen, L. Y.; Ou, C. C.; Lii, C. K. Dietary fat and garlic oil independently regulate hepatic cytochrome $\mathrm{P}_{450} 2 \mathrm{~B} 1$ and the placental form of glutathione $S$ transferase expression in rats. J. Nutr. 2001, 131, 1438-1443.

(23) Singh, G.; Kapoor, I. P.; Singh, P.; de Heluani, C. S.; de Lampasona, M. P.; Catalan, C. A. Chemistry, antioxidant and antimicrobial investigations on essential oil and oleoresins of Zingiber officinale. Food Chem. Toxicol. 2008, 46, 3295-3302.

(24) Bhopale, K. K.; Wu, H.; Boor, P. J.; Popov, V. L.; Ansari, G. A.; Kaphalia, B. S. Metabolic basis of ethanol-induced hepatic and pancreatic injury in hepatic alcohol dehydrogenase deficient deer mice. Alcohol 2006, 39, 179-188.

(25) Donohue, T. M.; Curry-McCoy, T. V.; Todero, S. L.; White, R L.; Kharbanda, K. K.; Nanji, A. A.; Osna, N. A. L-Buthionine (S,R) sulfoximine depletes hepatic glutathione but protects against ethanolinduced liver injury. Alcohol.: Clin. Exp. Res. 2007, 31, 1053-1060.

(26) Bradford, B. U.; O'Connell, T. M.; Han, J.; Kosyk, O.; Shymonyak, S.; Ross, P. K.; Winnike, J.; Kono, H.; Rusyn, I. Metabolomic profiling of a modified alcohol liquid diet model for liver injury in the mouse uncovers new markers of disease. Toxicol. Appl. Pharmacol. 2008, 232, 236-243.

(27) Gao, H.; Guan, T.; Li, C.; Zuo, G.; Yamahara, J.; Wang, J.; Li, Y. Treatment with ginger ameliorates fructose-Induced fatty liver and hypertriglyceridemia in rats: modulation of the hepatic carbohydrate response element-binding protein-mediated pathway. Evidence-Based Complement. Altern. Med. 2012, 570948.

(28) El-Sharaky, A. S.; Newairy, A. A.; Kamel, M. A.; Eweda, S. M. Protective effect of ginger extract against bromobenzene-induced hepatotoxicity in male rats. Food Chem. Toxicol. 2009, 47, 1584-1590.

(29) Yemitan, O. K.; Izegbu, M. C. Protective effects of Zingiber officinale (Zingiberaceae) against carbon tetrachloride and acetaminophen-induced hepatotoxicity in rats. Phytother. Res. 2006, 20, 9971002.

(30) Lu, K. H.; Liu, C. T.; Raghu, R.; Sheen, L. Y. Therapeutic potential of Chinese herbal medicines in alcoholic liver disease. $J$. Tradit. Complement. Med. 2012, 2, 115-122.

(31) Raghu, R.; Liu, C. T.; Tsai, M. H.; Tang, X.; Kalari, K. R.; Subramanian, S.; Sheen, L. Y. Transcriptome analysis of garlic induced hepatoprotection against alcoholic fatty liver. J. Agric. Food Chem. 2012, 60, 11104-11119.

(32) Williamson, J. R.; Scholz, R.; Browning, E. T.; Thurman, R. G.; Fukami, M. H. Metabolic effects of ethanol in perfused rat liver. J. Biol. Chem. 1969, 244, 5044-5054.
(33) Bakker, J. Blood lactate levels. Curr. Opin. Crit. Care. 1999, 5, 234-238.

(34) Bleyman, M. A.; Thurman, R. G. Comparison of acute and chronic ethanol administration on rates of ethanol elimination in the rat in vivo. Biochem. Pharmacol. 1979, 28, 2027-2030.

(35) Ji, S.; Lemasters, J. J.; Christenson, V.; Thurman, R. G. Periportal and pericentral pyridine nucleotide fluorescence from the surface of the perfused liver: evaluation of the hypothesis that chronic treatment with ethanol produces pericentral hypoxia. Proc. Natl. Acad. Sci. U.S.A. 1982, 79, 5415-5419.

(36) Yuki, T.; Thurman, R. G. The swift increase in alcohol metabolism. Time course for the increase in hepatic oxygen uptake and the involvement of glycolysis. Biochem. J. 1980, 186, 119-126.

(37) Li, S.; Liu, H.; Jin, Y.; Lin, S.; Cai, Z.; Jiang, Y. Metabolomics study of alcohol-induced liver injury and hepatocellular carcinoma xenografts in mice. J. Chromatogr., B 2011, 879, 2369-2375.

(38) Fernando, H.; Bhopale, K. K.; Kondraganti, S.; Kaphalia, B. S.; Ansari, G. A. S. Lipidomic changes in rat liver after long-term exposure to ethanol. Toxicol. Appl. Pharmacol. 2011, 255, 127-137.

(39) Lian, J. S.; Liu, W.; Hao, S. R.; Guo, Y. Z.; Huang, H. J.; Chen, D. Y.; Xie, Q.; Pan, X. P.; Xu, W.; Yuan, W. X.; Li, L. J.; Huang, J. R. A serum metabonomic study on the difference between alcohol- and $\mathrm{HBV}$-induced liver cirrhosis by ultraperformance liquid chromatography coupled to mass spectrometry plus quadrupole time-of-flight mass spectrometry. Chin. Med. J. 2011, 124, 1367-1373.

(40) Back, P.; Schernau-Potzi, L. Effect of ethanol on the metabolism of lithocholic acid in rat liver. Naunyn-Schmiedebergs Arch. Pharmacol. 1972, 275, 135-145.

(41) Haque, M. F.; Aghabeighi, B.; Wasil, M.; Hodges, S.; Harris, M. Oxygen free radicals in idiopathic facial pain. Bangladesh Med. Res. Counc. Bull. 1994, 20, 104-116.

(42) Kalhan, S. C.; Guo, L.; Edmison, J.; Dasarathy, S.; McCullough, A. J.; Hanson, R. W.; Milburn, M. Plasma metabolomic profile in nonalcoholic fatty liver disease. Metab.-Clin. Exp. 2011, 60, 404-413.

(43) Ahmed, M. H. Invasive and non-invasive investigations for nonalcoholic steatohepatitis (NASH): The need for biochemical markers. Scand. J. Gastroenterol. 2006, 41, 372. 